\title{
Terahertz and Thermal Testing of Glass-Fiber Reinforced Composites with Impact Damages
}

\author{
T. Chady, P. Lopato, and B. Szymanik \\ Department of Electrical and Computer Engineering, Faculty of Electrical Engineering, West Pomeranian University of Technology, \\ Al. Piastów 17, 70-310 Szczecin, Poland \\ Correspondence should be addressed to T. Chady, tchady@zut.edu.pl
}

Received 4 December 2011; Revised 3 February 2012; Accepted 6 February 2012

Academic Editor: Raimond Grimberg

Copyright (c) 2012 T. Chady et al. This is an open access article distributed under the Creative Commons Attribution License, which permits unrestricted use, distribution, and reproduction in any medium, provided the original work is properly cited.

\begin{abstract}
The studies on glass-fiber reinforced composites, due to their growing popularity and high diversity of industrial applications, are becoming an increasingly popular branch of the nondestructive testing. Mentioned composites are used, among other applications, in wind turbine blades and are exposed to various kinds of damages. The equipment reliability requirements force the development of accurate methods of their health monitoring. In this paper we present the study of composite samples with impact damages, using three methods: terahertz time domain inspection, active thermography with convective excitation, and active thermography with microwave excitation. The results of discrete Fourier transform of obtained time sequences of signals will be presented as well as some image processing of resulting amplitude and phase images. Proposed experimental methods combined with harmonic analysis are efficient tool of defects detection and allowed to detect flaws in examined specimens. Reader may find it interesting that in spite of differences in nature of applied experimental methods, one technique of signal processing (harmonic analysis) gave adequate and comparable results in each case.
\end{abstract}

\section{Introduction}

Polymer composite materials, because of their high strength to weight ratio and corrosion resistance, are more and more intensively used in various industries. One of the most important applications are hulls of ships and aircrafts, piping systems of liquid fuels, and wind turbine blades. All the mentioned structures are exposed to various environmental conditions, also mechanical impacts. The impact damages result in delaminations and significant weakening of the composite structure strength thus their detection is important issue. For this reason, impact damages should be evaluated using an appropriate technique. The ultrasonic testing, radiography, and shearography are common methods of composite materials nondestructive evaluation [1]. Because of nonconducting and nonmagnetic character of most polymer composites, a terahertz technique [2] can be applied in order to detect impact defects. Authors of [3] use time gating and peak value estimation to detect impact-induced delaminations. In case of our specimens, the response of defect is too weak to utilize this method. However, a harmonic analysis proved to be more sensitive and allowed to detect minute delaminations. Also in [3], frequency-gated harmonic analysis was successfully applied to evaluation of Kevlar/Nomex honeycomb sandwich panel. In [4], similar processing scheme with the same results is utilized for transmission measurements. In this paper, some basic information about $\mathrm{THz}$ time domain inspection system will be presented. Then, the harmonic analysis of THz signal will be done using the results of glass-fiber-reinforced composites reflection arrangement inspection.

In the second part of this paper, the basics of active thermography with convection heating excitation and with microwave excitation will be presented. The pulse-phase thermography with discrete Fourier transform of thermogram sequences [5] will be applied to examine the glass-fiberreinforced composite specimens. Some image processing of obtained amplitude images and phaseograms will be shown as well. 
S1:
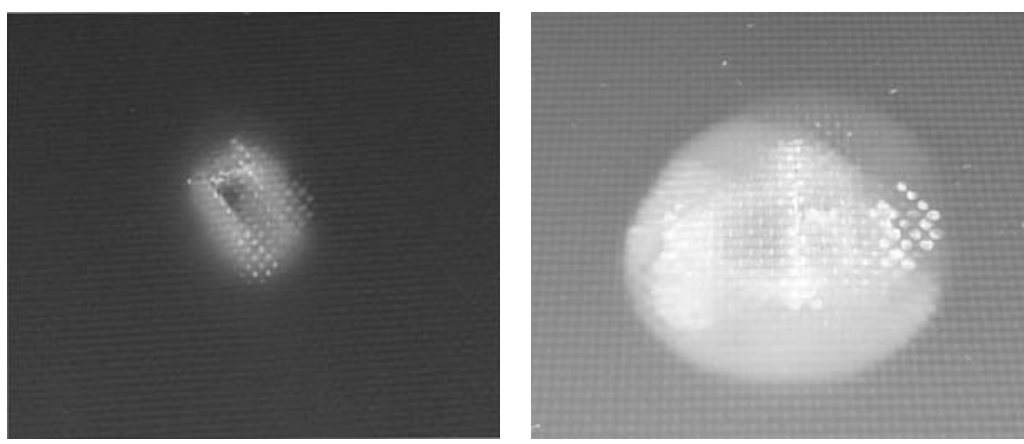

S2:
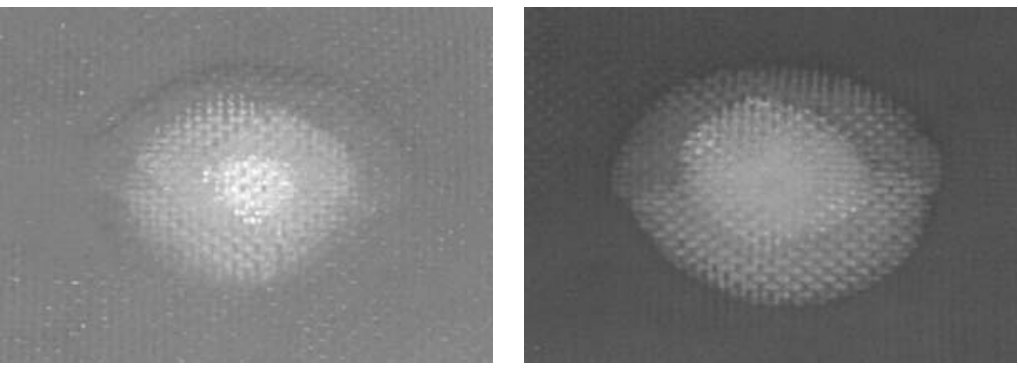

S3:
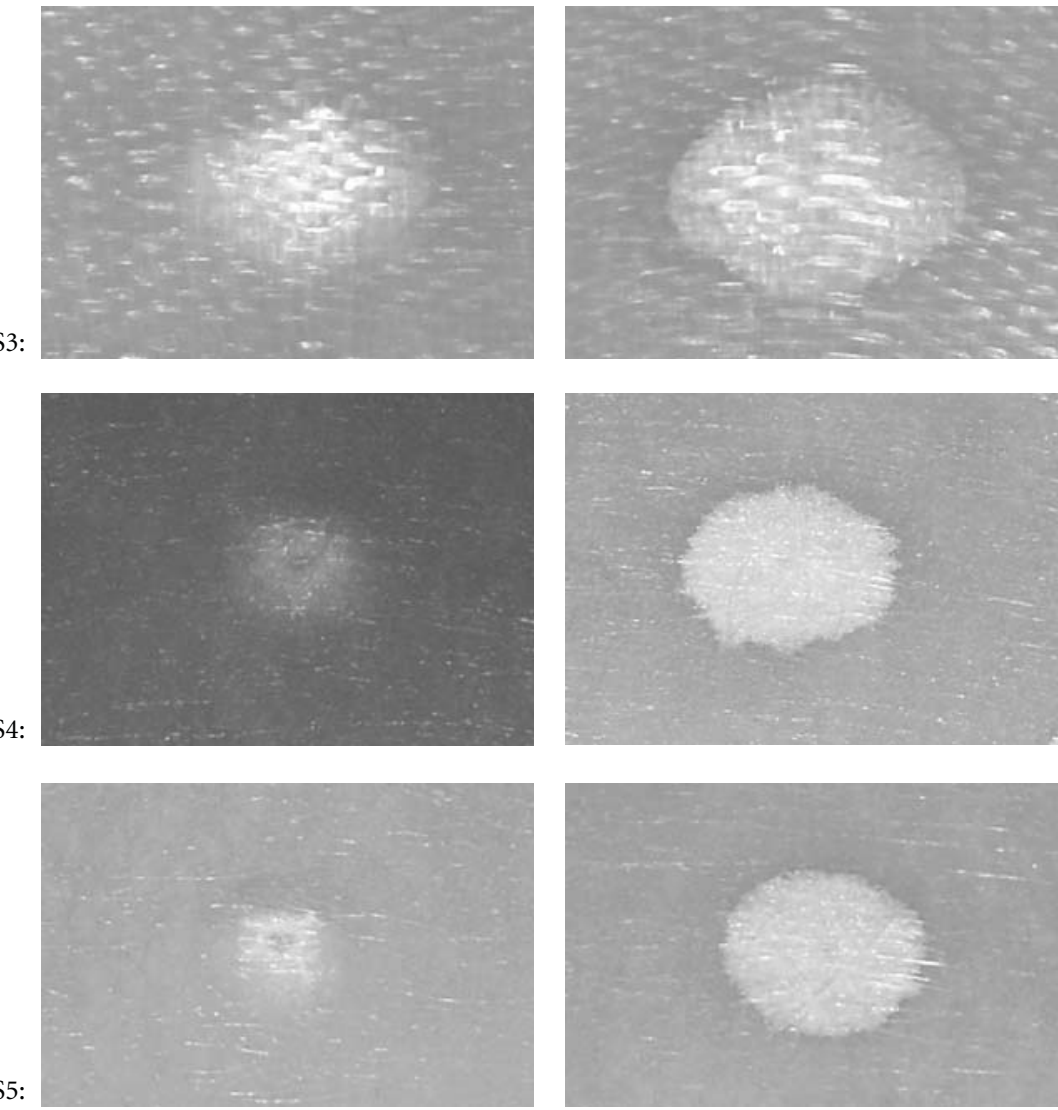

(a)

(b)

FIgURE 1: Photos of utilized impact damages in various glass-fibre-reinforced composite materials. Left: impact source side, right: opposite side. 


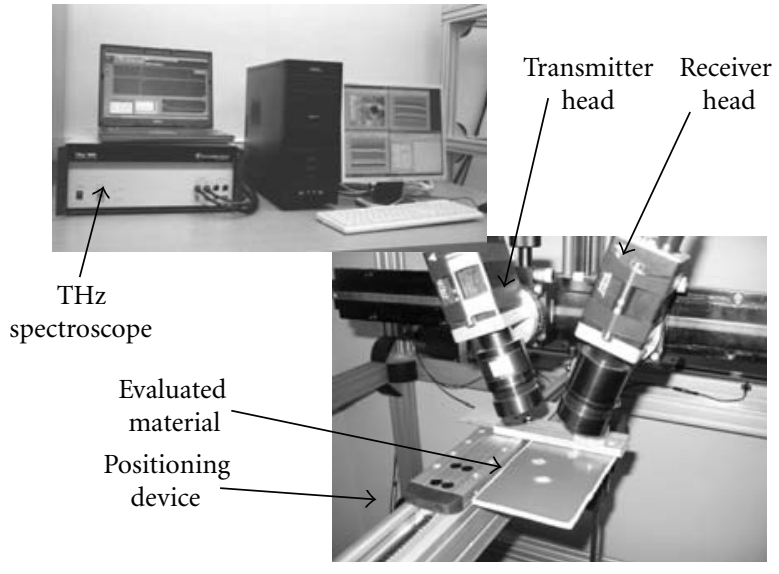

(a)

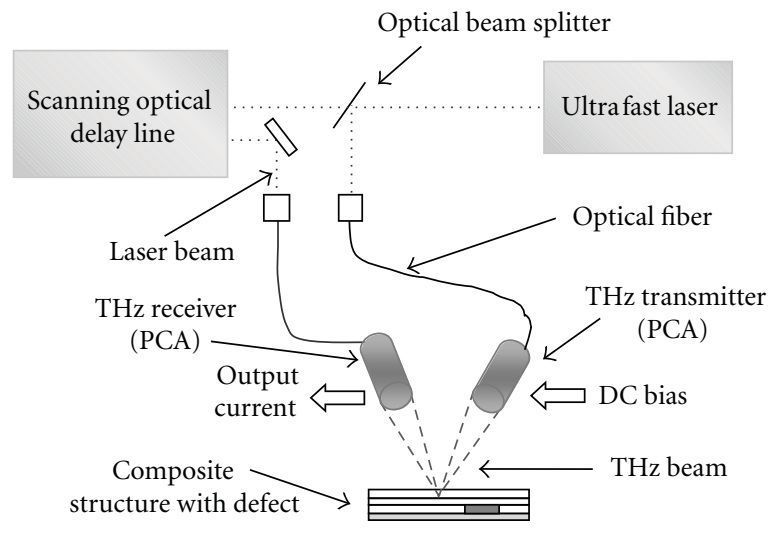

(b)

FIGURE 2: Photo (a) and simplified scheme (b) of pulsed terahertz measuring system.

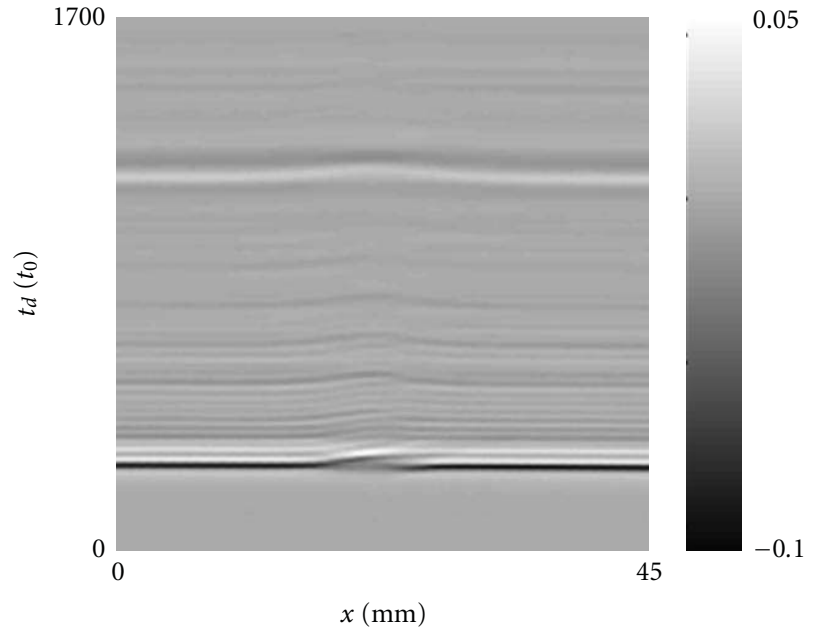

(a)

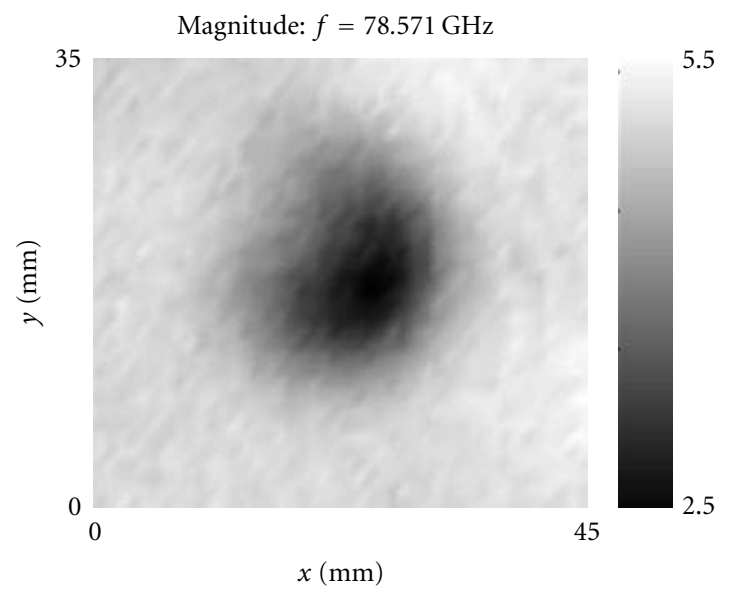

(c)

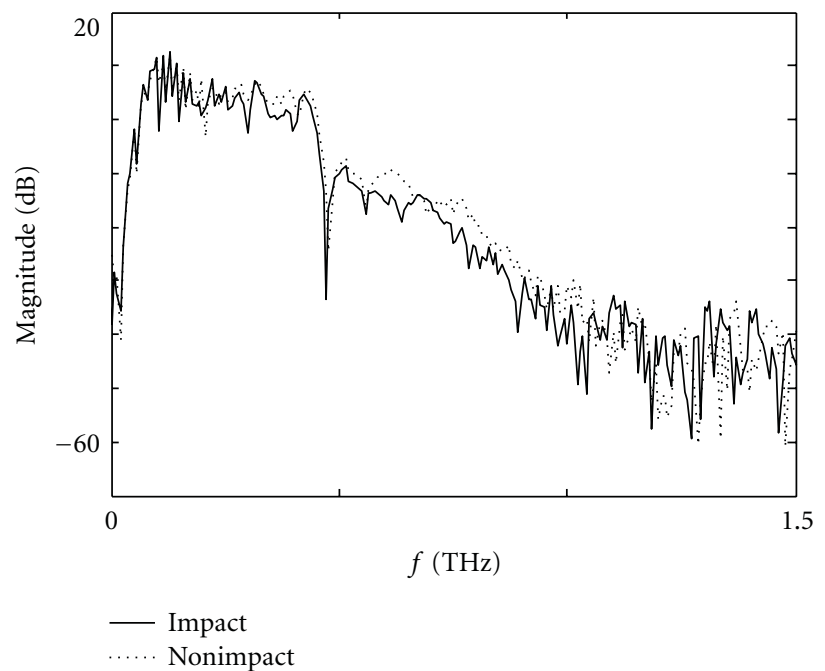

(b)

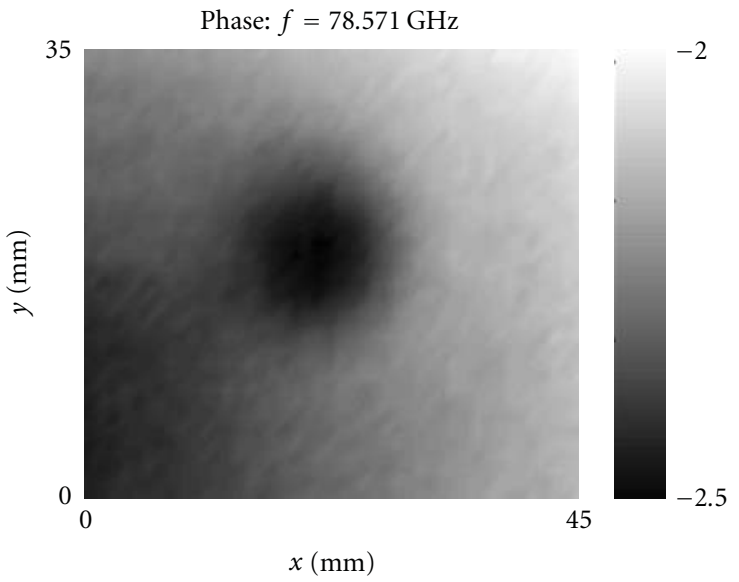

(d)

FIGURE 3: Results of S1 sample THz inspection: (a) raw B-scan signal, (b) frequency response of damaged and healthy material, (c) spatial distribution of measured waveform's magnitude in case of selected frequency, (d) distribution of phase. 


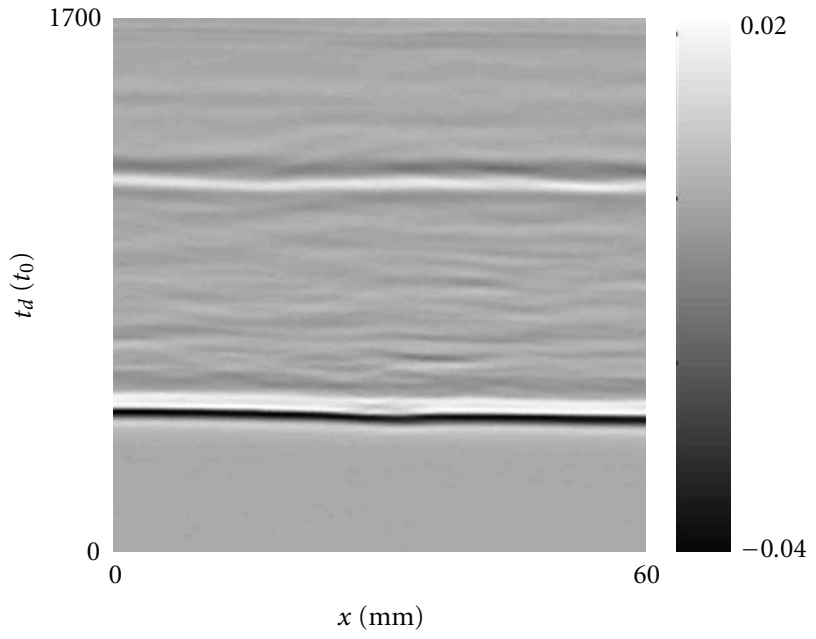

(a)

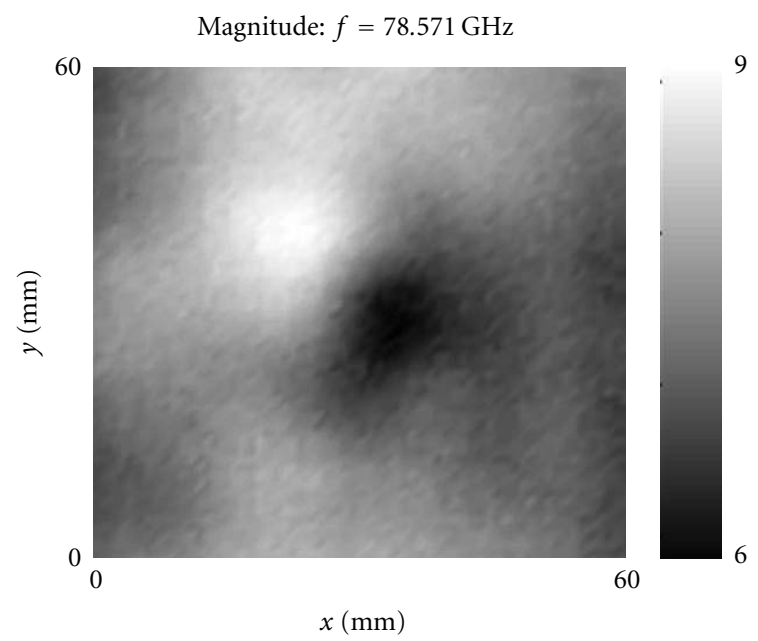

(c)

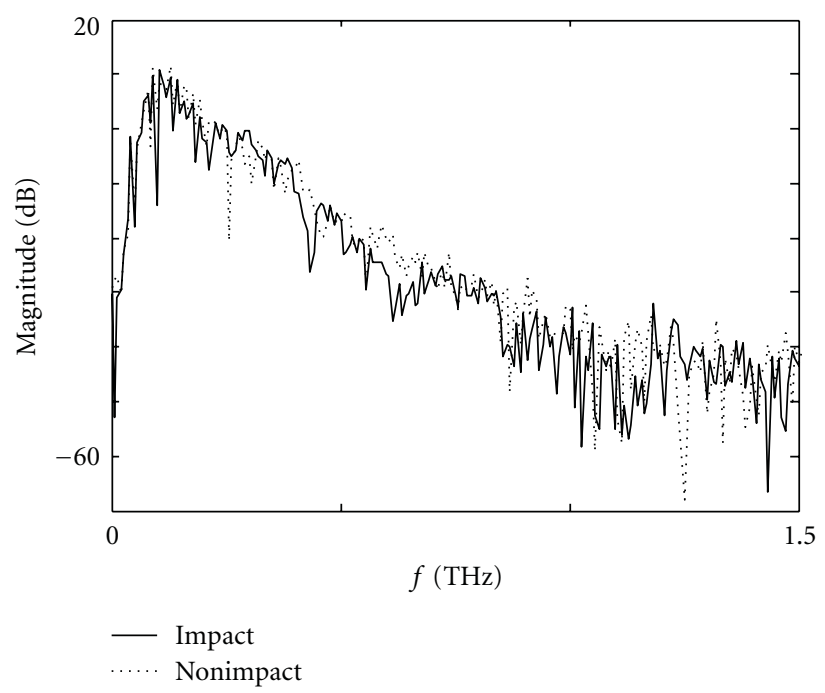

(b)

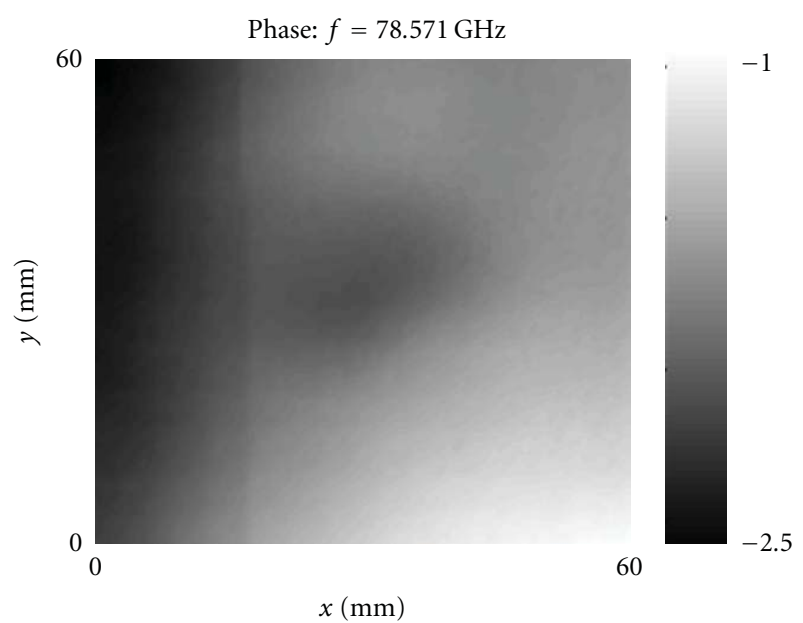

(d)

FIgURE 4: Results of S2 sample THz inspection: (a) raw B-scan signal, (b) frequency response of damaged and healthy material, (c) spatial distribution of measured waveform's magnitude in case of selected frequency, (d) distribution of phase.

\section{Examined Composite Materials}

Samples utilized in our experiments are presented in Figure 1. There are five types of glass-fiber-reinforced materials. Sample $S 1$ is a commercially available material which consists of 26 plies of glass fiber fabric with regular oriented fibers. The rest of samples are dedicated materials designed and manufactured mainly for presented tests. In case of all samples, a polyester resin Polimal 109-32 K was utilized. Sample S2 consists of 26 plies of glass roving fabric with density of $170 \mathrm{~g} / \mathrm{m}^{2}$ and orientation setup $\left[0^{\circ} / 90^{\circ}\right]_{13}$. Sample S3 consists of 10 plies of glass roving fabric with density of $430 \mathrm{~g} / \mathrm{m}^{2}$ and orientation setup $\left[0^{\circ} / 90^{\circ}\right]_{5}$. Sample $S 4$ consists of 6 plies of glass mat Vetrotex Unifilo 4750-138 with density of $450 \mathrm{~g} / \mathrm{m}^{2}$. The reinforcement of sample $\mathrm{S} 5$ is the same as in case of S4, but the resin was doped with synthetic rubber Hypro VTBNX 1300x33. All the composites were made by a hand layup method. After polymer matrix curing, the polymer plates were cut out. The sample's thickness in each case was equal to $5 \mathrm{~mm}$. The impact of 16 Joules energy was made with spherical-shaped impactor of $2 \mathrm{~g}$ mass.

\section{Terahertz Time Domain Inspection}

Pulsed terahertz NDT system based on the Tray-4000 spectroscope of Picometrix and its simplified scheme is presented in Figure 2. The main components of the system are a pair of photoconductive and fiber coupled transducers (transmitter and receiver), an ultra-fast laser, and an optical delay line.

The photoconductive antenna (PCA) based transmitter and receiver heads work in a reflection mode (as shown in Figure 2). An ultra-short $\mathrm{THz}$ pulse generated by the transmitting PCA is focused on an evaluated materials surface, reflected and picked up by the receiving PCA. On the basis of achieved signals, it is possible to obtain information 


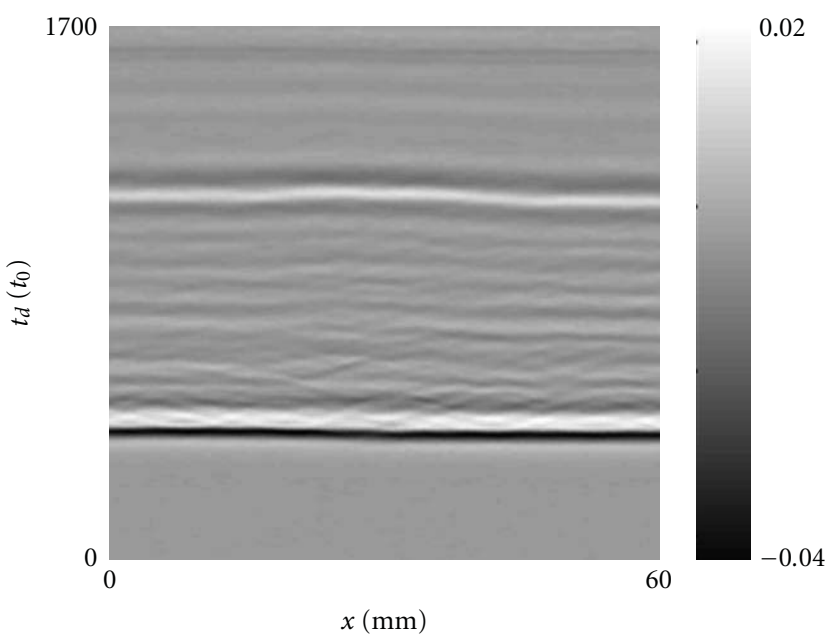

(a)

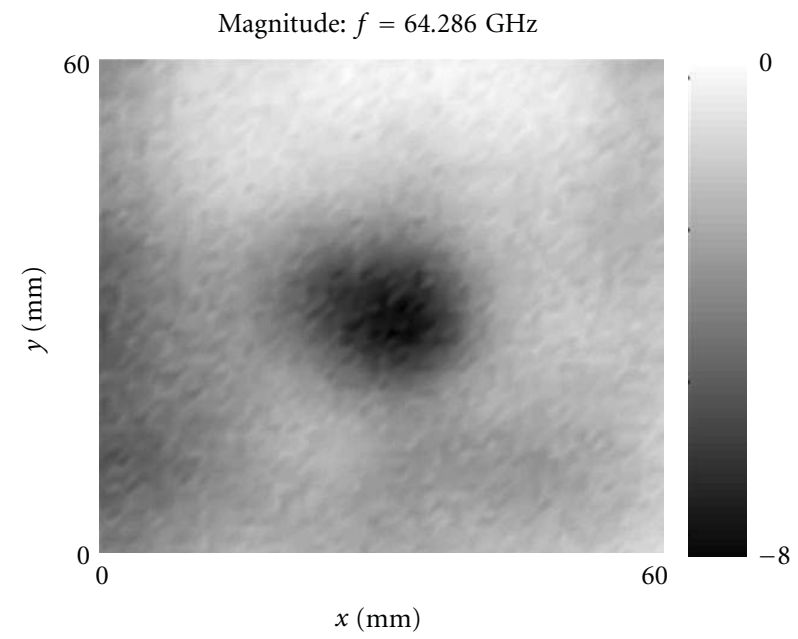

(c)

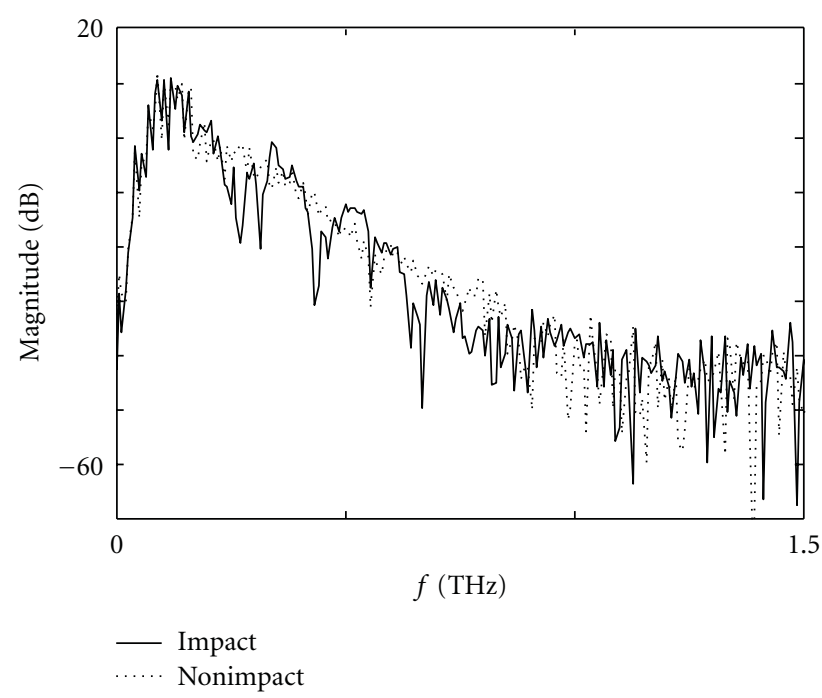

(b)

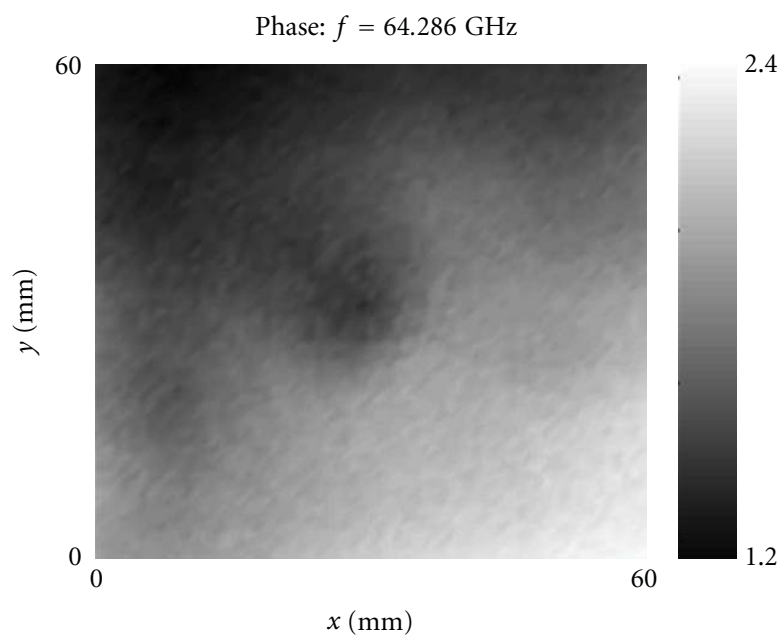

(d)

Figure 5: Results of S3 sample THz inspection: (a) raw B-scan signal, (b) frequency response of damaged and healthy material, (c) spatial distribution of measured waveform's magnitude in case of selected frequency, (d) distribution of phase.

about internal structure of the evaluated object (similar as in the ultrasonic testing). It is possible to detect any defect which disturbs distribution of refractive index, for example, inclusion, delamination, void, material inhomogeneities (fiber/matrix distribution), and internal interfaces between layers (in layered structures). Main advantages associated with terahertz technique are

(i) noncontact measurement in reflection and transmission arrangement,

(ii) nonionizing nature,

(iii) inner structure and spectral information is obtainable,

(iv) fraction of millimeter resolution.

Main disadvantages of terahertz technique are

(i) low power of $\mathrm{THz}$ emitters, (ii) low speed of examination (need of raster scanning),

(iii) restriction to nonconductive materials (because of high frequency and skin effect).

\section{Terahertz Time Domain Experiment's Regime and Results}

The impact damaged samples were examined using pulsed terahertz technique in reflection arrangement as it was shown in Figure 2. Exemplary inspection results (B-scan signals) are presented in Figures 3(a)-7(a) (in case of all samples S1-S5). For each measuring point $(x, y)$, a time domain response waveform consisting of 1792 samples was acquired. Sampling time step $t_{0}$ was equal to $78.1 \mathrm{fs}$, thus the resulting range of time delay in case of all measurements was $140 \mathrm{ps}$. Because of nature of the excitation, the response consists of pulses also. Two main pulses correspond to front 


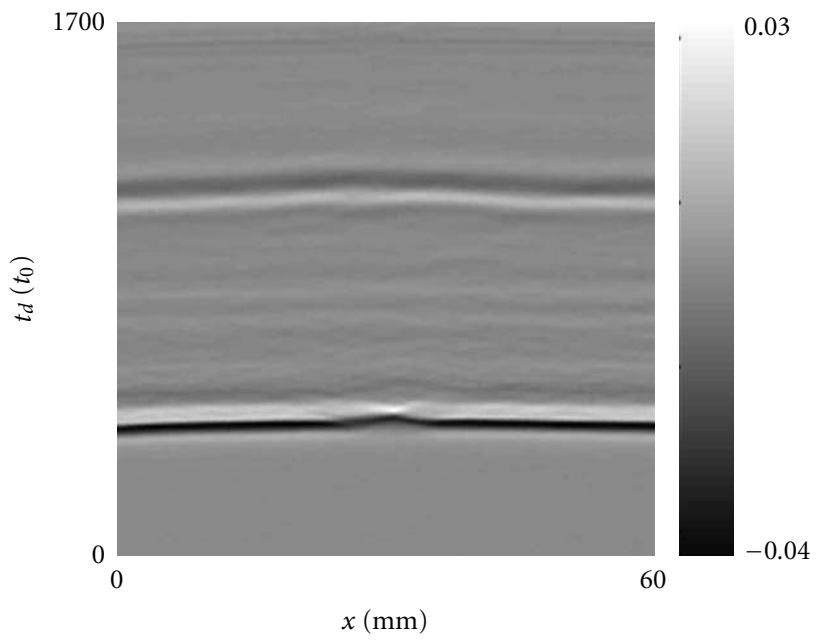

(a)

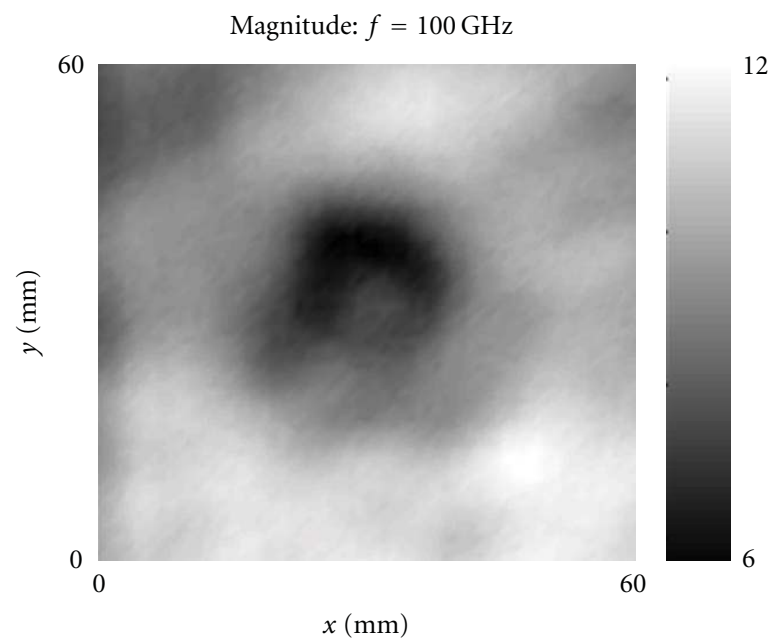

(c)

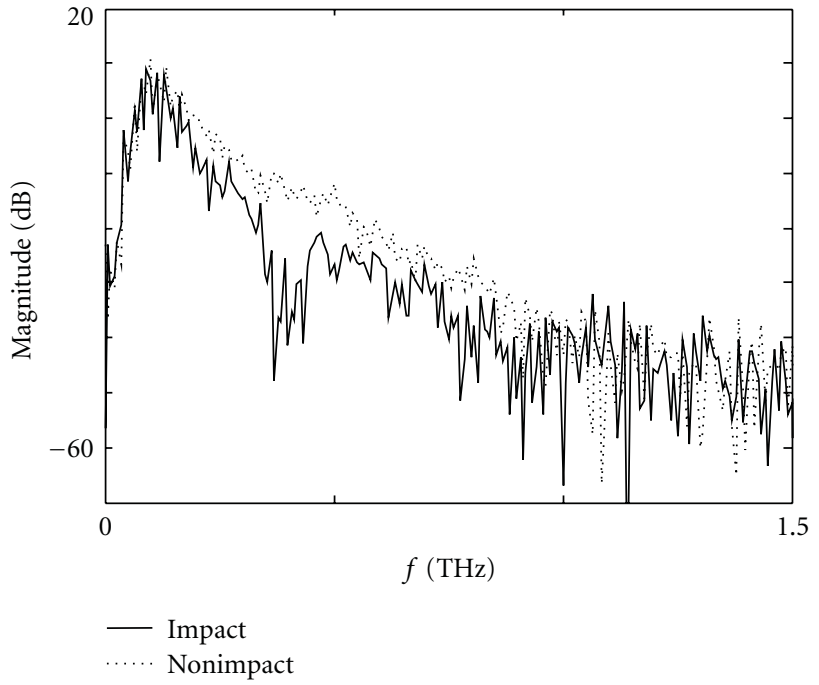

(b)

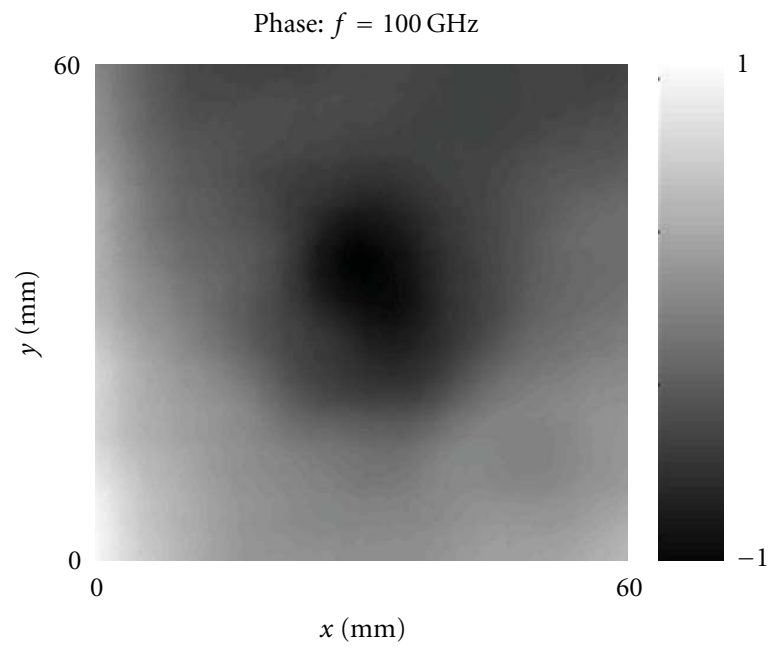

(d)

Figure 6: Results of S4 sample THz inspection: (a) raw B-scan signal, (b) frequency response of damaged and healthy material, (c) spatial distribution of measured waveform's magnitude in case of selected frequency, (d) distribution of phase.

(FSR) and back (BSR) surface reflections. One can observe FSR's in presented B-scans close to $t_{d}=500$ and BSR's in the vicinity of $t_{d}=1250$. Any additional pulse is caused by internal structure reflections. In case of layered materials (samples S1, S2, and S3), because of noticeable difference of resin and fabric refractive indices in terahertz domain, the interfaces between the layers are clearly visible directly in B-scan. The place where the surface material was exposed to mechanical forcing can be deformed upon impact. This surface deformation acts as a lens and causes local increase in amplitude of FSR. This effect is observable especially in case of samples S4 and S5. An influence of impact is clearly visible when inner structure (layers) are distinguishable. In B-scan of S1 and S3 samples, change of position of the inner layers and BSR can be seen directly. In case of other materials such visual analysis of measured signals is not sufficient.
We propose harmonic analysis of terahertz signals in order to obtain an information about impact damage position. Before this, all measured signals were median filtered in time domain and processed by Fourier transform. Frequency responses of damaged and healthy materials are presented and compared in Figures 3(b)-7(b) (in case of all samples S1-S5). The biggest differences between damaged and healthy areas are in $0.3-0.9 \mathrm{THz}$ range. They are mainly associated with close-to-front surface changes in material and can be utilized in order to localize surface damage. Lower frequencies enable estimation of position and shape of impact caused defect in vicinity of back surface of the evaluated material. Spatial distributions of measured waveform's magnitude and phase in case of lower frequencies are shown in Figures 3(c)-7(c) and Figures 3(d)-7(d). In all 


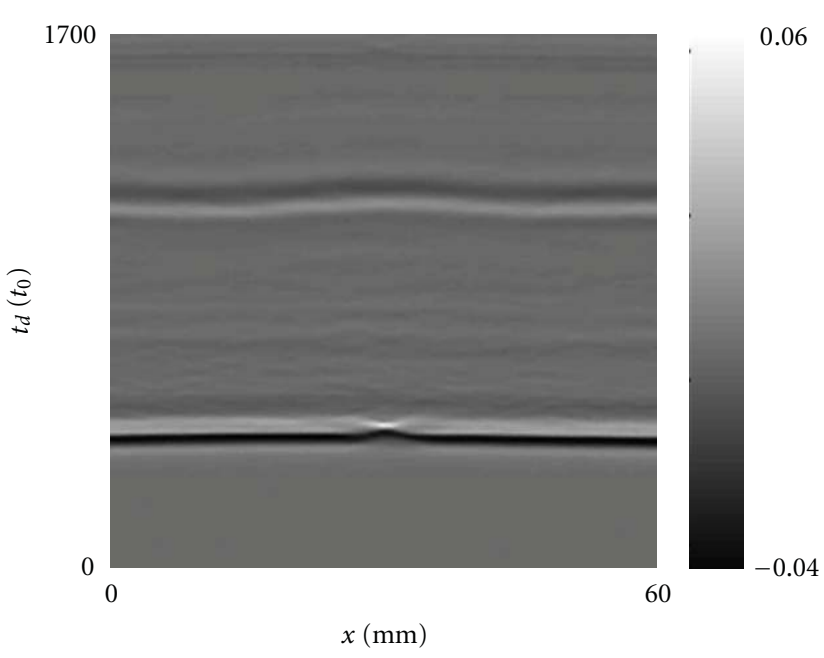

(a)

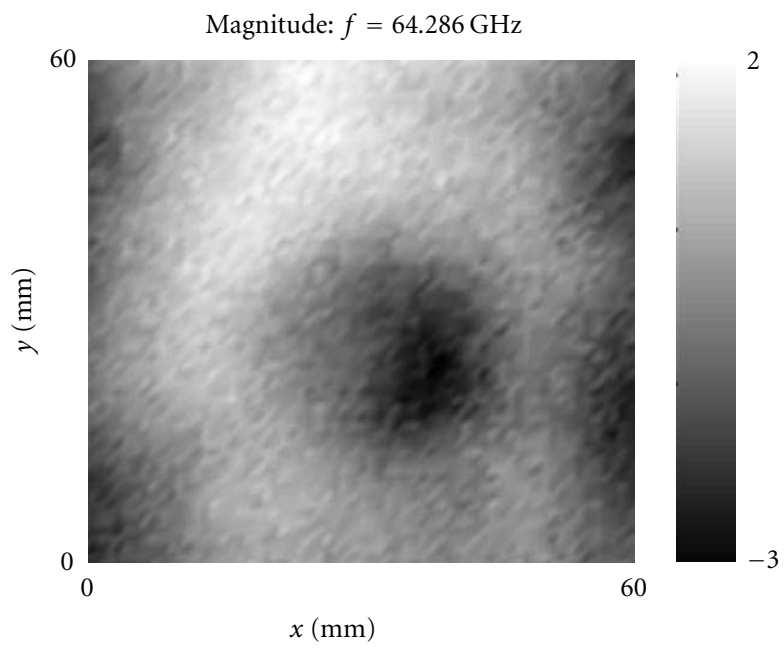

(c)



(b)

Phase: $f=64.286 \mathrm{GHz}$

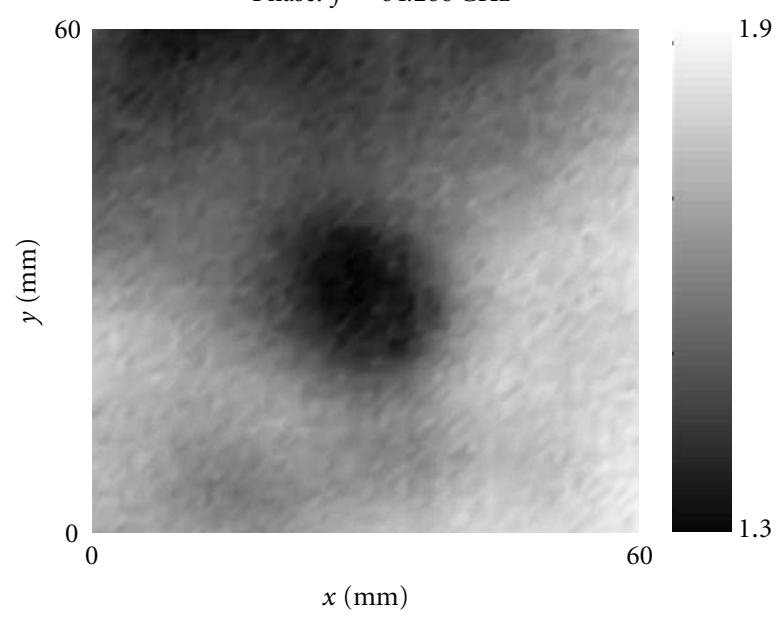

(d)

Figure 7: Results of S5 sample THz inspection: (a) raw B-scan signal, (b) frequency response of damaged and healthy material, (c) spatial distribution of measured waveform's magnitude in case of selected frequency, (d) distribution of phase.

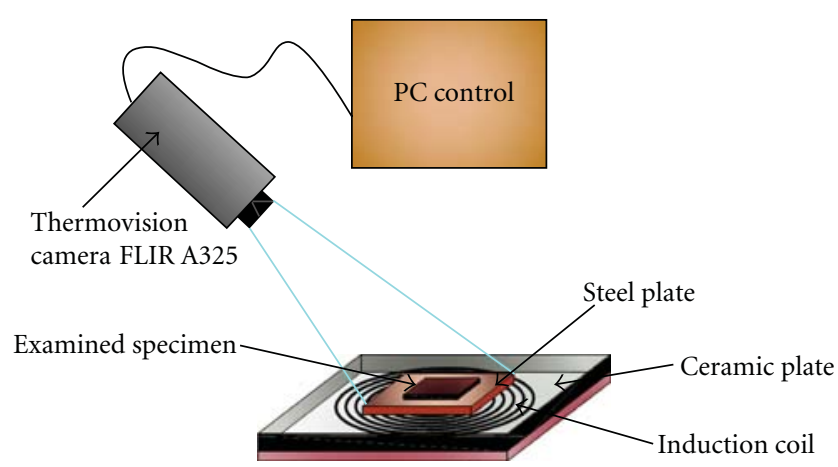

(a)

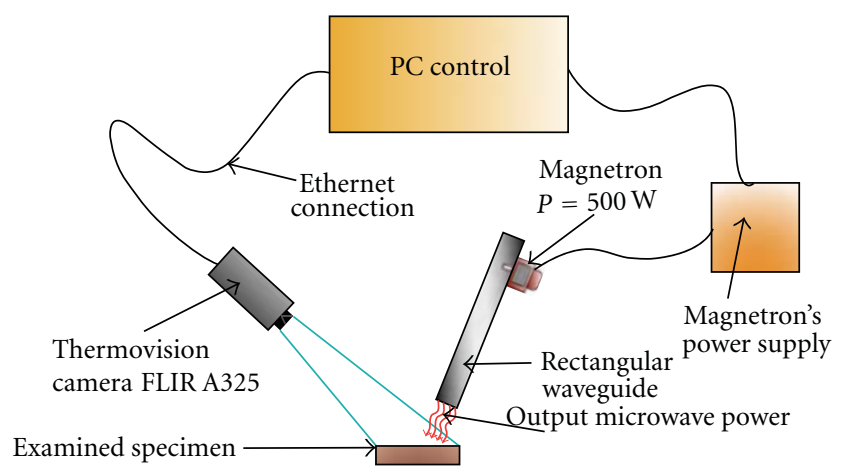

(b)

FIgURE 8: The method's scheme. (a) active thermography with convective excitation, (b) active thermography with microwave excitation. 


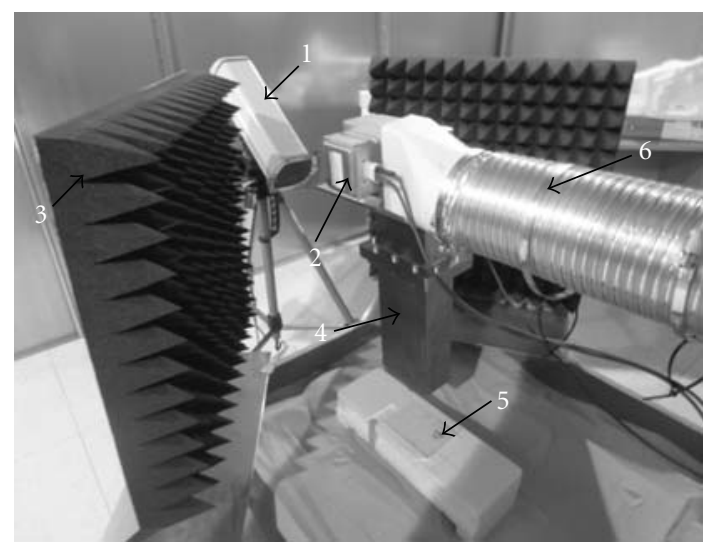

FIGURE 9: Experimental setup for active thermography with microwave excitation. (1) thermovision camera in protective housing, (2) magnetron, (3) absorbers, (4) rectangular waveguide, (5) examined sample, (6) magnetron's cooling system.

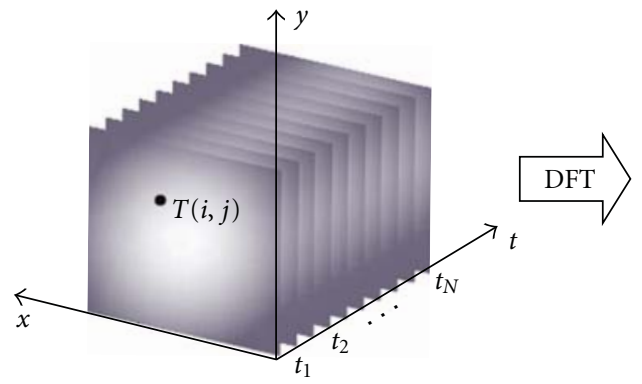

Thermogram sequence

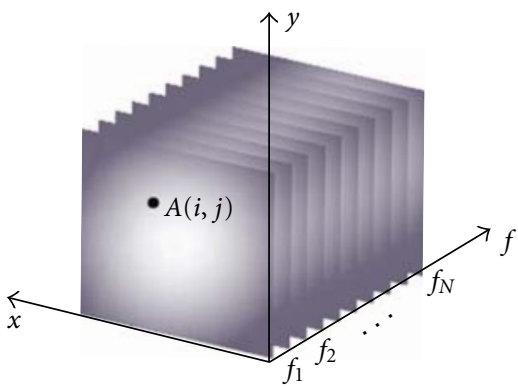

Amplitude sequence

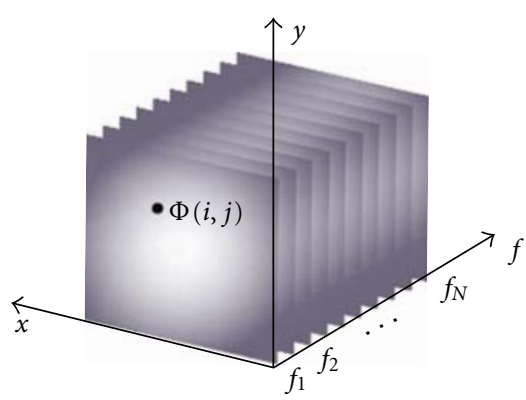

Phase sequence

Figure 10: The discrete Fourier transform for thermogram sequence scheme.

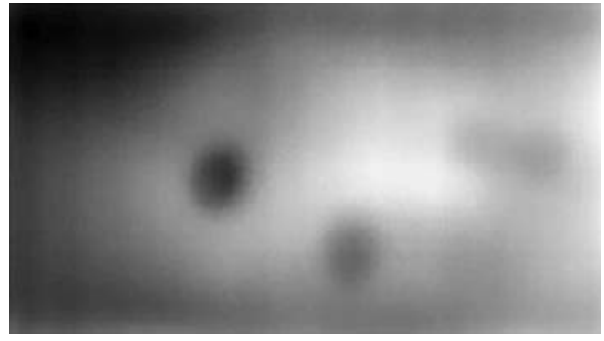

(a)



(c)

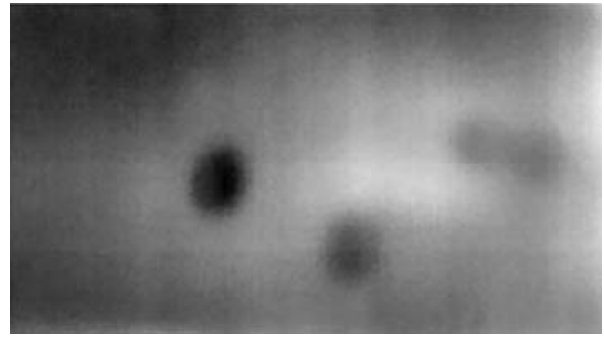

(b)

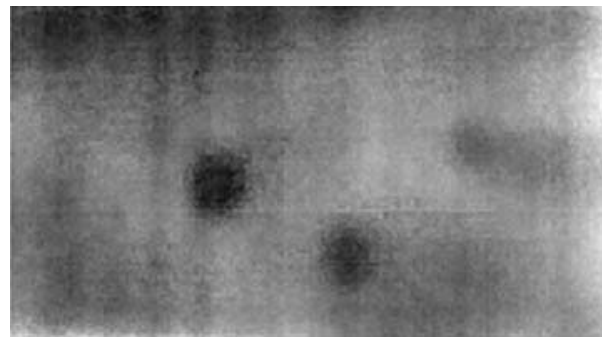

(d)

FIgURE 11: Obtained phaseograms for sample S1 (a) $0.018 \mathrm{~Hz}$, (b) $0.036 \mathrm{~Hz}$, (c) $0.054 \mathrm{~Hz}$, (d) $0.072 \mathrm{~Hz}$. 


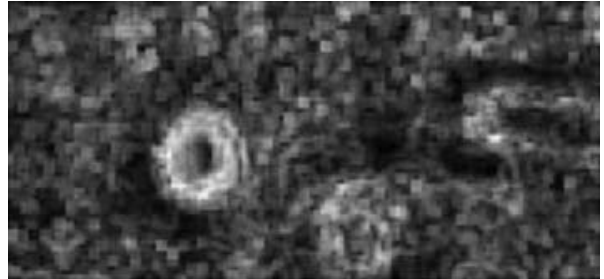

(a)

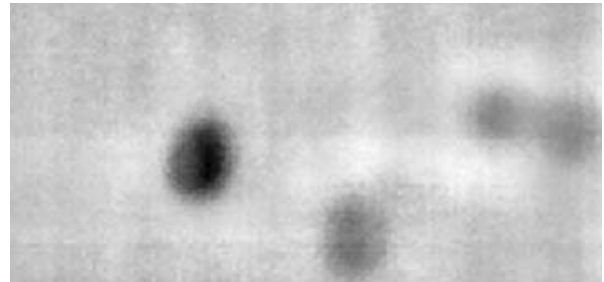

(b)

FIGURE 12: Image processing of chosen amplitude images and phaseograms for sample S1. (a) Amplitude image enhanced with median and standard deviation filtering, (b) phase enhanced with median filtering.

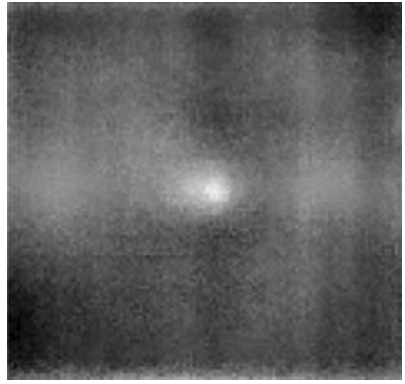

(a)



(b)

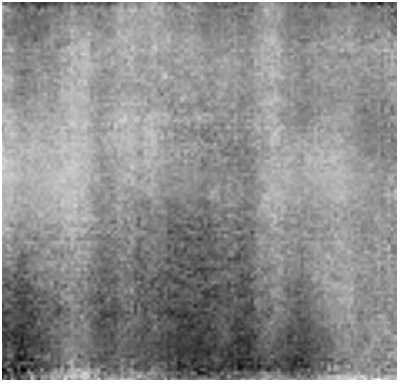

(c)

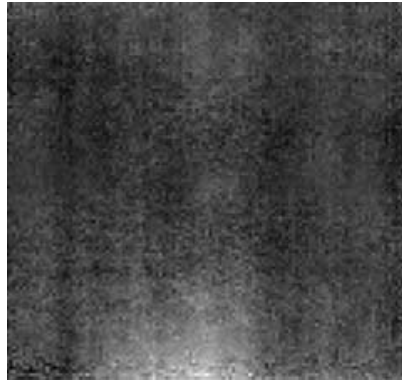

(d)

Figure 13: Obtained phaseograms for sample S2. (a) $0.018 \mathrm{~Hz}$, (b) $0.036 \mathrm{~Hz}$, (c) $0.054 \mathrm{~Hz}$, (d) $0.072 \mathrm{~Hz}$.

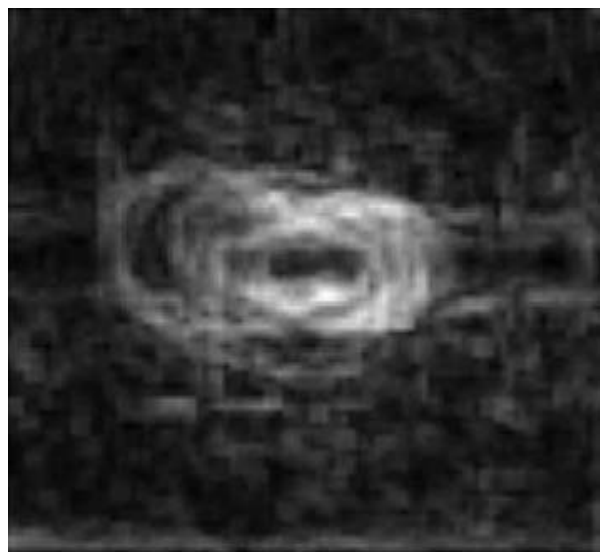

(a)

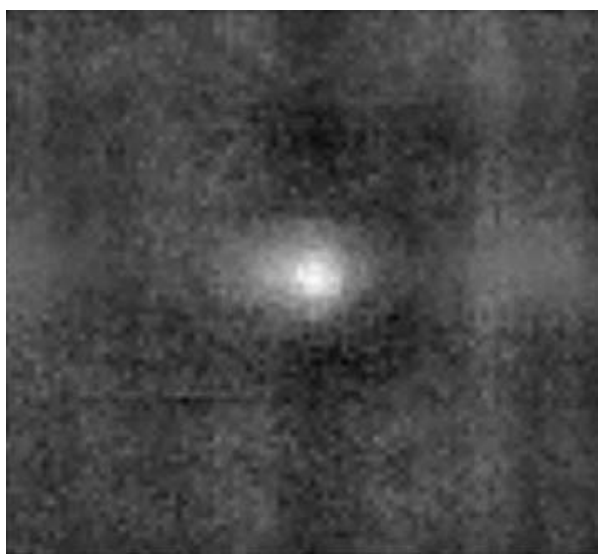

(b)

FIGURE 14: Image processing of chosen amplitude images and phaseograms for sample S2. (a) Amplitude image enhanced with median and standard deviation filtering, (b) phase enhanced with median filtering.

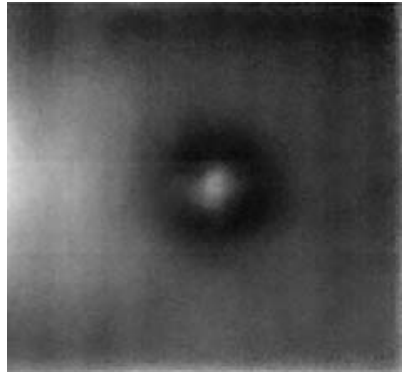

(a)

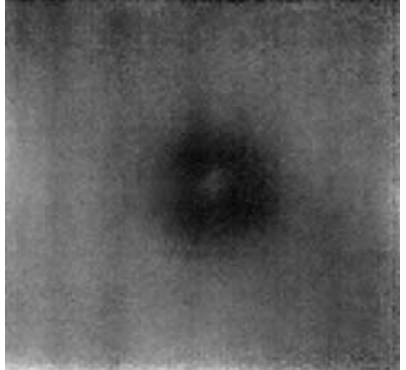

(b)

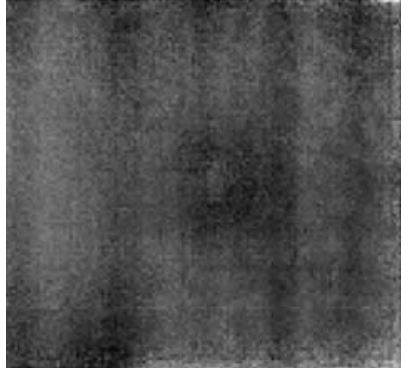

(c)

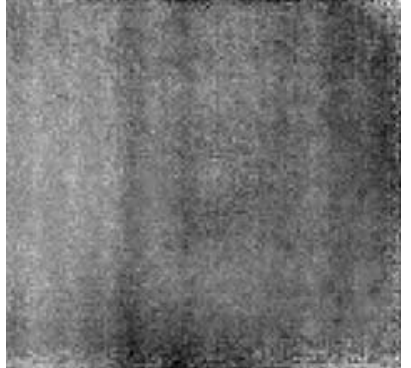

(d)

FIGURE 15: Obtained phaseograms for sample S3. (a) $0.018 \mathrm{~Hz}$, (b) $0.036 \mathrm{~Hz}$, (c) $0.054 \mathrm{~Hz}$, (d) $0.072 \mathrm{~Hz}$. 


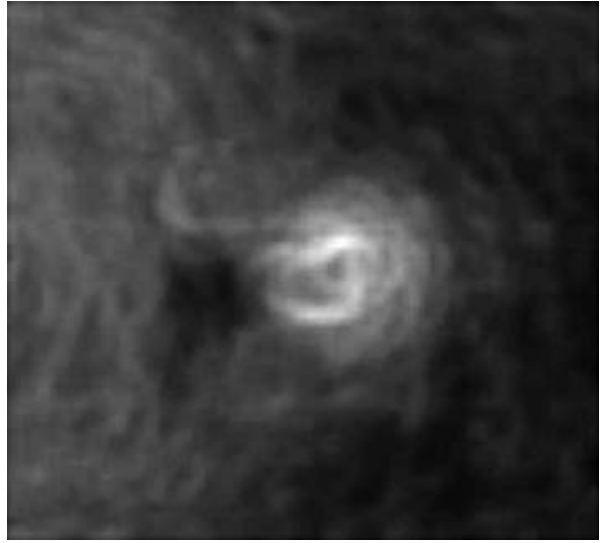

(a)

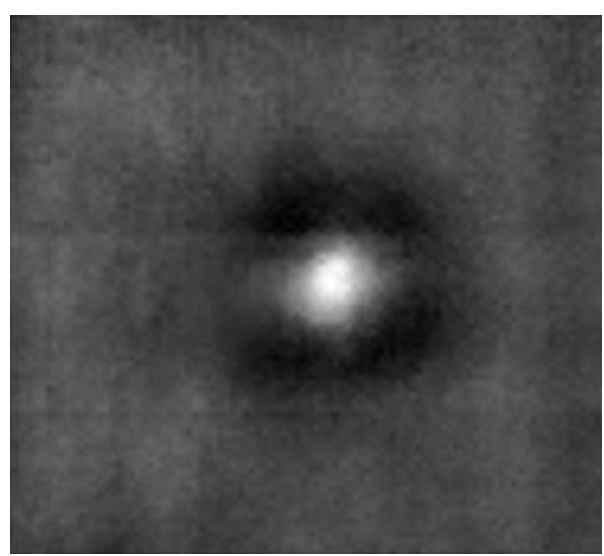

(b)

Figure 16: Image processing of chosen amplitude images and phaseograms for sample S3. (a) Amplitude image enhanced with median and standard deviation filtering, (b) phase enhanced with median filtering.



(a)

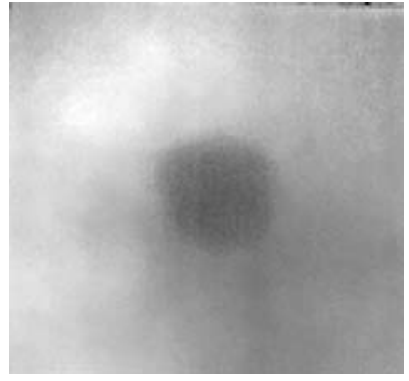

(b)

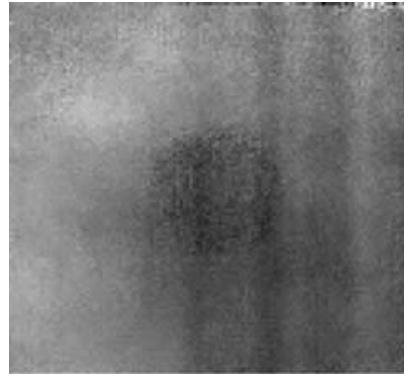

(c)

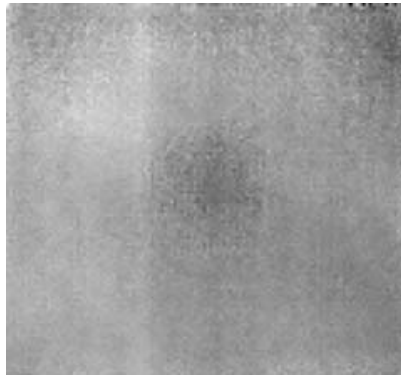

(d)

FIgURE 17: Obtained phaseograms for sample S4. (a) $0.018 \mathrm{~Hz}$, (b) $0.036 \mathrm{~Hz}$, (c) $0.054 \mathrm{~Hz}$, (d) $0.072 \mathrm{~Hz}$.

cases, localization of defect based on obtained distributions is possible.

In case of frequencies higher than $0.9 \mathrm{THz}$, because of very low SNR, signal contains no applicable information about defect (Figures 4, 5, and 6).

\section{Active Thermography with Convection and Microwave Excitation}

Composite samples with impact damages were investigated using active infrared thermography. As the excitation, we propose two energy sources: convective heat flow from inductively heated steel plate (contact method) and microwave heating (contactless method). In the convective heating, the composite sample is placed on the inductively heated steel plate (Figure $8(\mathrm{a})$ ). The free flow of heat through the examined specimen can be then observed using thermovision camera. Defects are detected as (depending on the damage type) under- or overheated spots. The main disadvantage of this method is the requirement that the sample should be in contact with the heated steel plate, which can make problems for practical applications.

The active thermography with microwave excitation is the contactless method. In this technique, examined sample is heated by high power microwaves $(500 \mathrm{~W}$, working at the frequency $2.45 \mathrm{GHz}$ ). Heating phase is observed by the properly secured thermovision camera. A schematic drawing of the method is shown in Figure 8(b) whereas Figure 9 shows the designed laboratory setup.

Since in both techniques the heating phase can be observed, the pulsed phase thermography (PPT) could be applied. This method combines the experimental procedure used in pulsed thermography (PT), with signal analysis used in modulated thermography (MT) [6]. The thermograms' sequence is recorded, while the heat pulse is applied to examined specimen.

The analysis of obtained sequence is based on discrete Fourier transform (DFT), which allows to evaluate the output as the combination of phase and amplitude. The procedure scheme is presented in Figure 10.

The well-known Fourier Transform of each pixel in the thermogram sequence may be written as follows [7]:

$$
F_{n}=\sum_{k=0}^{N-1} T(k) e^{-j 2 \pi i n k / N}=\operatorname{Re}_{n}+\operatorname{Im}_{n}
$$

where $n$ denotes the frequency increment, and Re and Im indicate the real and imaginary parts of transform. The 


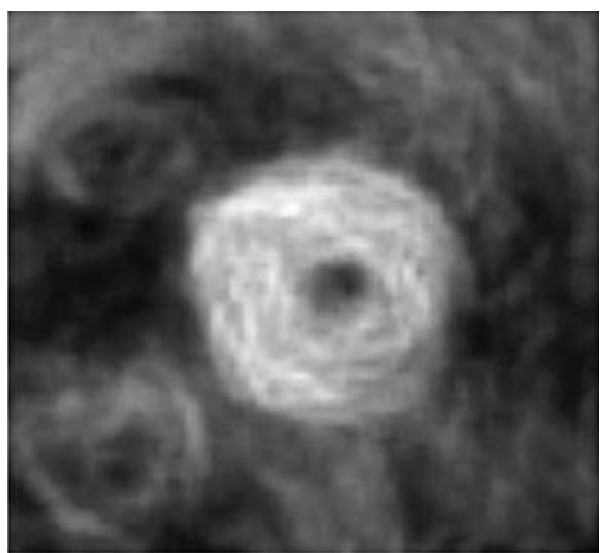

(a)

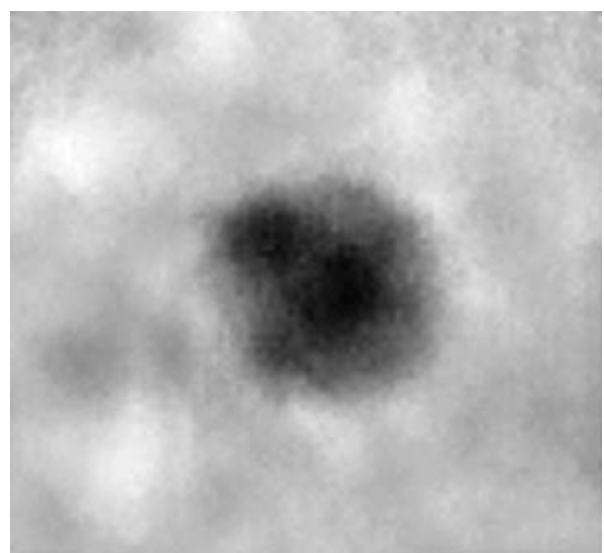

(b)

FIGURE 18: Image processing of chosen amplitude images and phaseograms for sample S4. (a) Amplitude image enhanced with median and standard deviation filtering, (b) phase enhanced with median filtering.

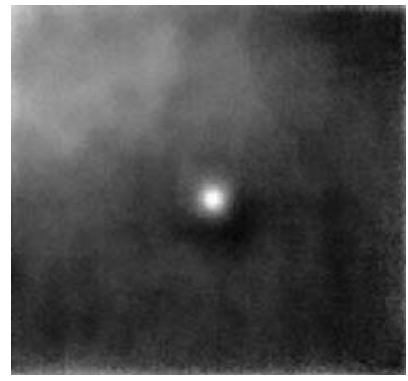

(a)

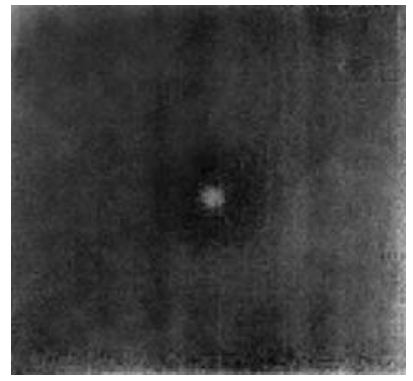

(b)

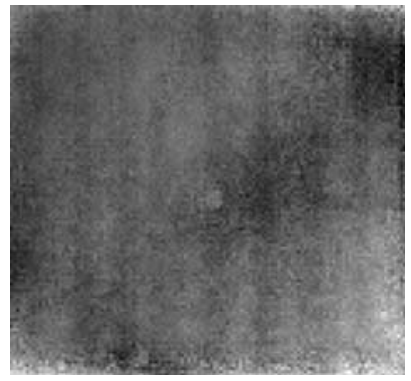

(c)

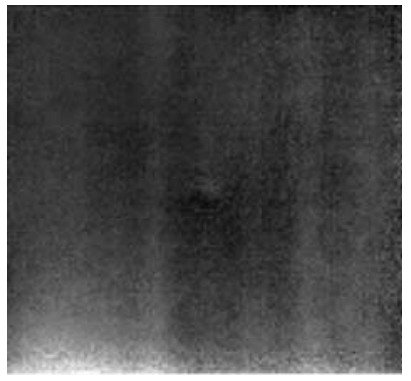

(d)

Figure 19: Obtained phaseograms for sample S5. (a) $0.018 \mathrm{~Hz}$, (b) $0.036 \mathrm{~Hz}$, (c) $0.054 \mathrm{~Hz}$, (d) $0.072 \mathrm{~Hz}$.

amplitude $\left(A_{n}\right)$ and phase $\left(\phi_{n}\right)$ are computed using following formulas:

$$
A_{n}=\sqrt{\operatorname{Re}_{n}^{2}+\operatorname{Im}_{n}^{2}}, \quad \Phi_{n}=\arctan \frac{\operatorname{Im}_{n}}{\operatorname{Re}_{n}} .
$$

Both amplitude images and phaseograms were used to obtain reliable results of specimens' evaluation.

\subsection{Active Thermography with Convection Heating Experi-} ment's Regime and Results. In case of convection heating, all five samples were tested, using the same heating time and recording frequency. The observation time was set to 55 second, and recording frequency was $9 \mathrm{~Hz}$, which allowed us to obtain 495 thermograms in one sequence. For every sample, the same procedure of signal processing was used: the DFT of thermograms sequence was performed, chosen amplitude images and phaseograms were then processed using median or standard deviation filter, to enhance the contrast between the background and defect.

The results (chosen phaseograms, and image processing of selected amplitude images and phaseograms) are shown in Figures 11-20.

It can be notice that best results for phase images of samples S2-S5 (Figures 13(a), 15(a), 17(a), and 19(a)) are obtained for frequency of $0.018 \mathrm{~Hz}$, which was simultaneously the lowest available frequency in these experiments. In case of sample S1, the phaseogram for frequency of $0.036 \mathrm{~Hz}$ presents all the defects in most distinguishable way (Figure 11(b)). The median (for heating unevenness removal) and standard deviation (for defect enhancement) filtering of signal amplitude image gives in each case the information of defect location and size.

\subsection{Active Thermography with Microwave Heating Exper-} iment's Regime and Results. Microwave-enhanced infrared thermography is a relatively new NDT method. Using microwaves as the energy source gives a possibility of volumetric heating of the material, which can significantly speed up the heat process. Moreover, this method is contactless. Unfortunately, the high power microwaves, needed to obtain visible temperature differences between the defect and the background, may cause damage to the thermovision camera. Therefore, additional protective housing is needed in this case. Special metallic mesh, used as camera lens protection, increases the noise level in obtained thermograms. The image processing of thermogram sequence is then more demanding and time consuming.

In case of microwave heating the observation time was set to 100 seconds, and recording frequency was set 


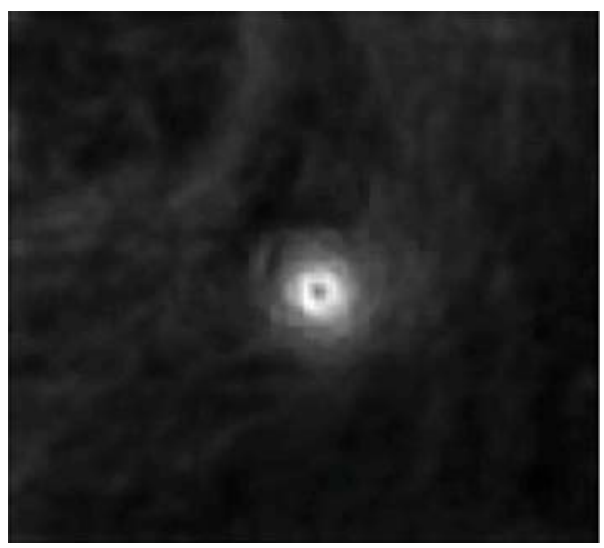

(a)

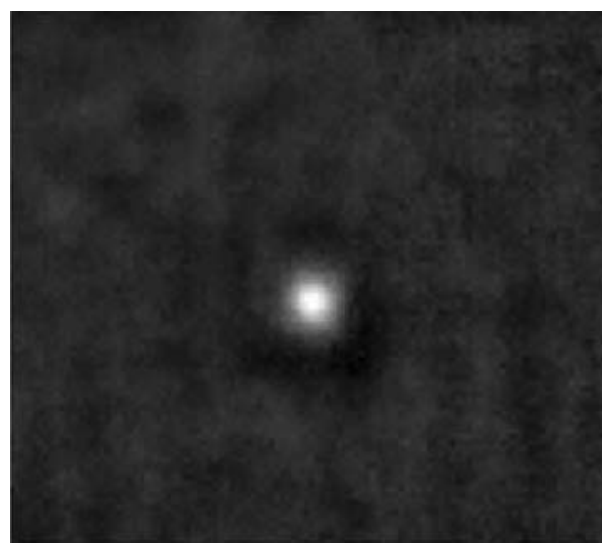

(b)

FIGURE 20: Image processing of chosen amplitude images and phaseograms for sample S5. (a) Amplitude image enhanced with median and standard deviation filtering, (b) phase enhanced with median filtering.

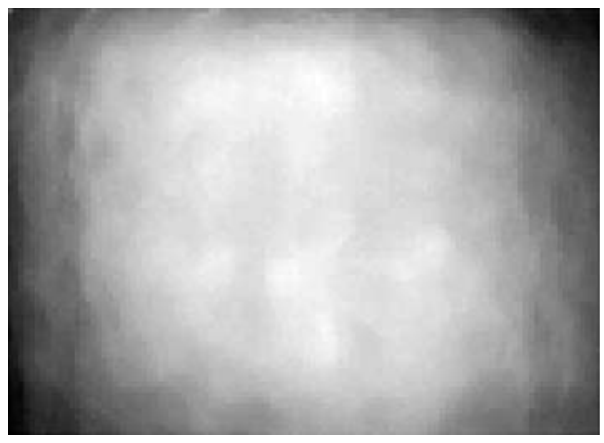

(a)

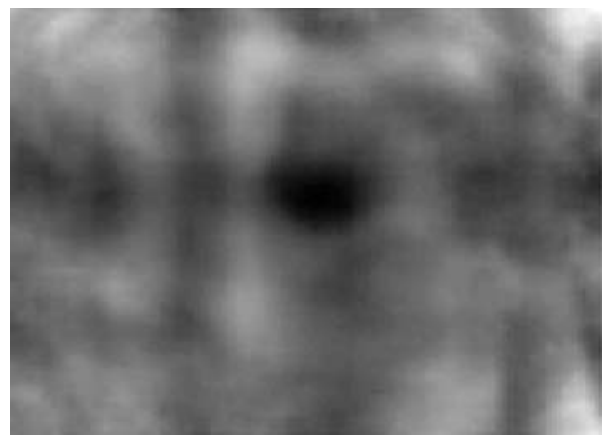

(b)

FIGURE 21: Obtained results for sample S2. (a) Chosen initial amplitude image, (b) amplitude image after several filtering procedures.

to $15 \mathrm{~Hz}$, which allowed to obtain 1500 thermograms in one sequence. After the DFT procedure, chosen amplitude images were enhanced using procedure based on multiply filtering. Obtained results (only for samples S2 to S5) are promising (Figures 14-17), but obviously future work is needed to obtain more accurately outcome. Heating of sample S1 and obtained thermogram sequence analysis using DFT did not allow for damage detection, therefore, it was omitted in the presentation of the resulting images (Figures 21-24).

The image processing of available amplitude images was based on median filtering and contrast enhancement. Obtained results give the defect approximate location, but the information about flaws' size is not contained in resulting images.

Due to high noise level in the output thermograms, the research on improving the methodology of measurement should be continued. The time of heating extension in order to increase the temperature contrast between the background and defect as well as the sequence of thermograms recording frequency increment is, therefore, considered.

\section{Conclusions}

Both utilized methods of glass-fiber-reinforced composites examination enable detection of impact caused defects. The pulsed $\mathrm{THz}$ technique offers very wide and unique (compared to other common methods) abilities of inspection: high resolution, no need to use any additional coupling medium, availability of spectroscopic information, and finally a defect depth information is also provided. Simple harmonic analysis is sufficient tool in detection of damages caused by mechanical impacts in case of various kinds of materials.

Active infrared thermography is a fast (sometimes it allows real time monitoring of structures) and giving tangible results method. The convection excitation allows obtaining information about the location and size of the defect. The harmonic analysis of obtained thermograms' sequences for specimens S1-S5 (representing different types of composite material which may be found in practical usage) proved to be sufficient to obtain reliable results. In each case, the defect itself is clearly visible, moreover, additional analysis using standard devaition filtration, allowed 


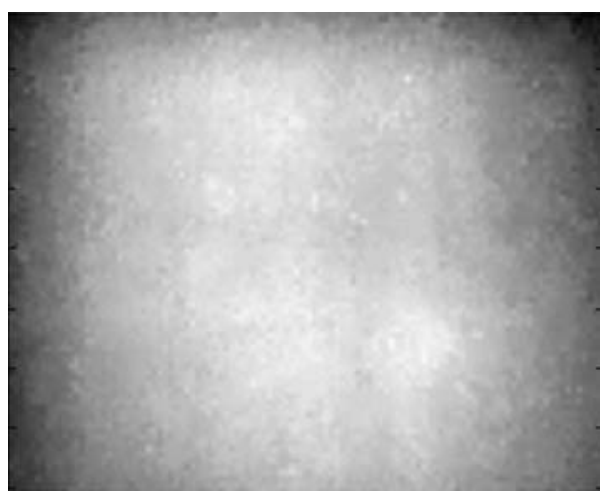

(a)

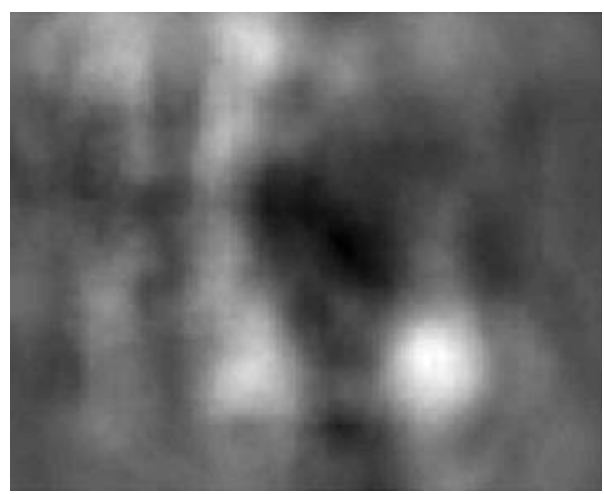

(b)

FIGURE 22: Obtained results for sample S3. (a) Chosen initial $t$ amplitude image, (b) amplitude image after several filtering procedures.

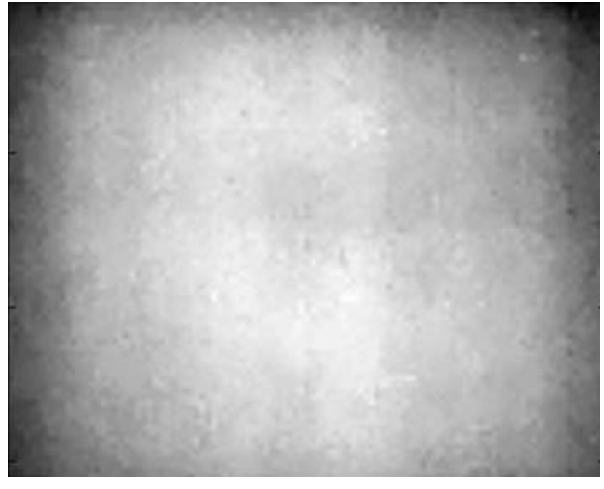

(a)

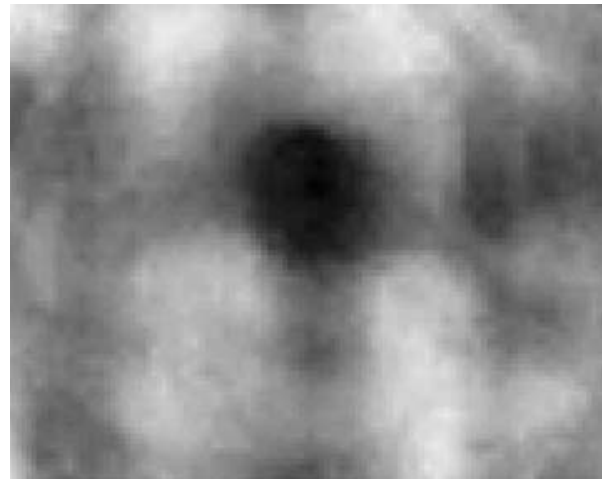

(b)

FigURE 23: Obtained results for sample S4. (a) Chosen initial amplitude image, (b) amplitude image after several filtering procedures.



(a)

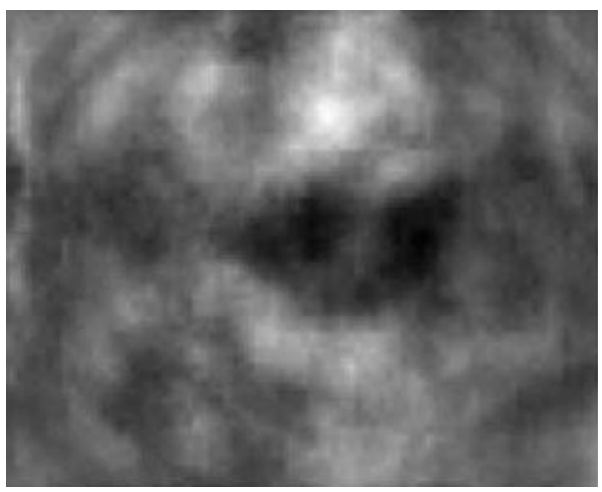

(b)

FIGURE 24: Obtained results for sample S5. (a) Chosen initial amplitude image, (b) amplitude image after several filtering procedures. 
to visualize delamination arose in the vicinity of damage. The application of this method in practice, however, due to the fact that it requires contact with the heat source, can be sometimes difficult. The microwave excitation, on the other hand, is a contactless method. However, it requires additional thermovision camera protection, which causes significant increment of obtained thermograms noise level. Therefore, the received thermogram sequence image processing is much more difficult. The harmonic analysis in this case was connected with additional signal processing involving trend removal based on median filtering. Nevertheless, obtained results allow only to an approximate localization of the defect. The further development of the active infrared thermography with microwave excitation method, however, is highly warranted because of the ease of its industrial application, high speed, and the ability to simultaneous study of materials' large surfaces.

Each sample was examined using two different methods (i.e., THz imaging and active thermography with two energy sources), but the signal processing technique was chosen to be the same for data obtained with both utilized methods. Reader may notice that harmonic analysis allowed to detect the flaws in examined samples, and obtained results may be used to quantitative and qualitative evaluation of materials.

\section{Acknowledgment}

This work was supported in part by European Commission project HEMOW: Health Monitoring of Offshore Wind Farms (reference: FP7-PEOPLE-2010-IRSES-GA-269202).

\section{References}

[1] A. Kapadia, Non Destructive Testing of Composite Materials, TWI Ltd.

[2] D. M. Mittleman, M. Gupta, R. Neelamani, R. G. Baraniuk, J. V. Rudd, and M. Koch, "Recent advances in terahertz imaging," Applied Physics B, vol. 68, no. 6, pp. 1085-1094, 1999.

[3] D. K. Hsu, K. H. Im, C. P. Chiou, and D. J. Barnard, "An exploration of the utilities of terahertz waves for the NDE of composites," in Proceedings of the Review of Quantitative Nondestructive Evaluation, vol. 30 of AIP Conference Proceedings, pp. 533-540, New York, NY, USA, 2011.

[4] F. Rutz, M. Koch, S. Khare, M. Moneke, H. Richter, and U. Ewert, "Terahertz quality control of polymeric products," International Journal of Infrared and Millimeter Waves, vol. 27, no. 4, pp. 547-556, 2006.

[5] X. Maldague and S. Marinetti, "Pulse phase infrared thermography," Journal of Applied Physics, vol. 79, no. 5, pp. 2694-2698, 1996.

[6] X. Maladegue, Theory and Practice of Infrared Technology for Nondestructive Testing, John Wiley and Sons, New York, NY, USA, 2001.

[7] S. Marinetti, Y. A. Plotnikov, W. P. Winfree, and A. Braggiotti, "Pulse phase thermography for defect detection and visualization," in Nondestructive Evaluation of Aging Aircraft, Airports, and Aerospace Hardware III, vol. 3586 of Proceedings of SPIE, pp. 230-238. 

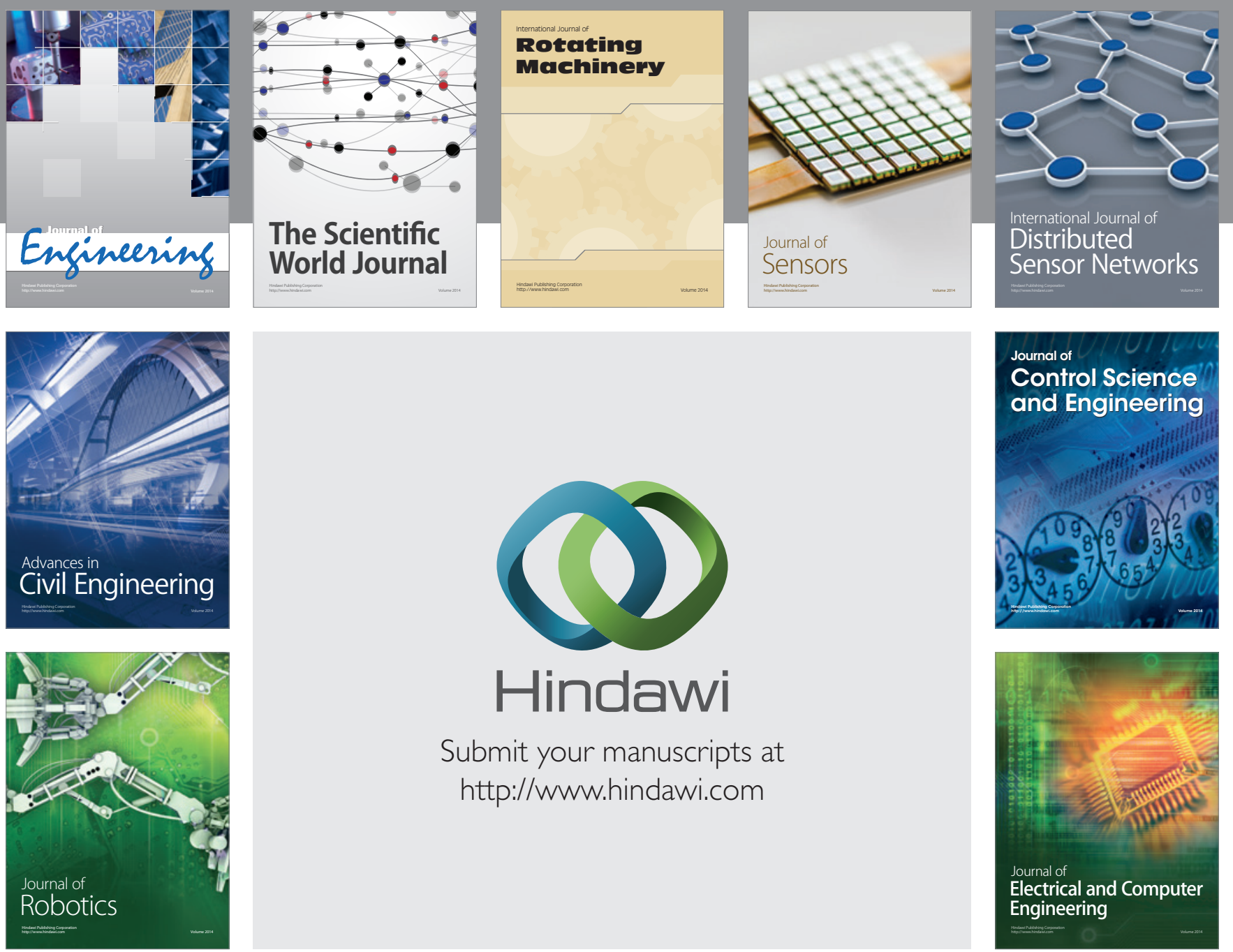

Submit your manuscripts at

http://www.hindawi.com
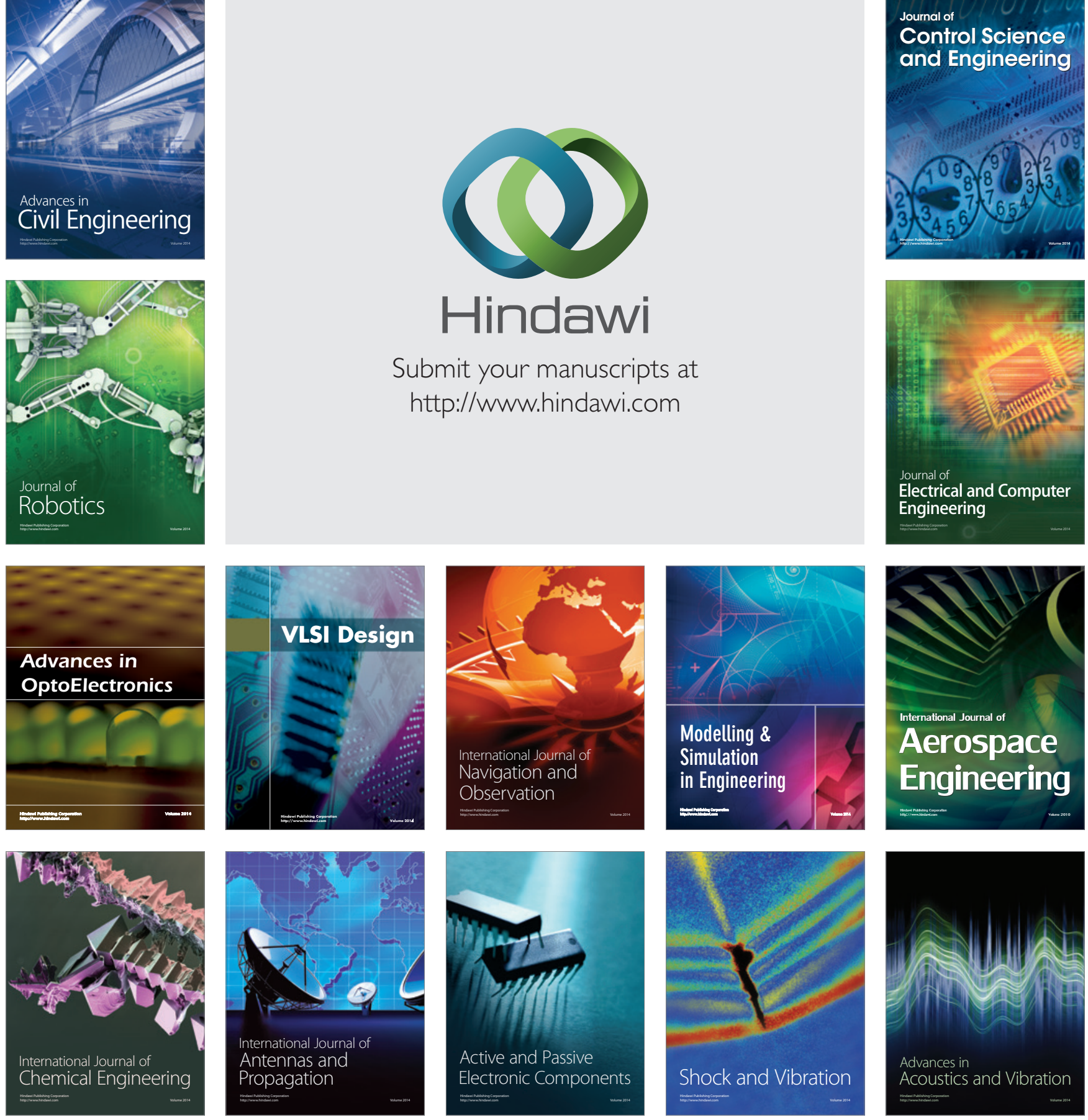